\title{
Inverse identification of unsteady heat transfer coefficient using infrared thermography in a fin an tube heat exchanger assembly
}

by M. Mobtil*, D. Bougeard*

\author{
* Mines Douai, El, F-59500 Douai, \\ France Univ Lille Nord de France, F-59000 Lille, France mohammed.mobtil@mines-douai.fr \\ * Mines Douai, El, F-59500 Douai, \\ France Univ Lille Nord de France, F-59000 Lille, France daniel.bougeard@mines-douai.fr
}

\begin{abstract}
An inverse method is used to determine the transient heat transfer coefficient distribution over the fin of the second row of a staggered finned tube heat exchanger assembly. The experimental test bench uses an infrared thermography system that record the temperature drop of the circular fin of the heat exchanger during a transient experiment. The unsteady temperature field is used in a specific inverse method in order to retrieve the heat transfer coefficient over the fin.
\end{abstract}

\section{Introduction}

High precision local heat transfer investigation can be achieved with transient techniques. These techniques have been widely used with simplified heat conduction models in order to find analytical expressions of heat transfer coefficient (HTC). For instance, in the lumped capacitance model, the material is considered being thermally thin and lateral heat conduction into the material is neglected [1]. With this model, only the elapsed time needed for the material to move from an initial temperature to a final one has to be evaluated. Other models with less restrictive hypotheses have been used by other authors. For instance, in [2] the material is supposed to be thermally thin but lateral conduction is not neglected. The method used the temporal evolution of the fin surface temperature recorded by IR thermography. Finally, no matter what model is used, the estimation of local HTC with transient techniques consists in measuring the temperature field of the body surface during a thermal transient state and a steady state fluid flow. The tacit hypothesis of transient methods is that heat transfer coefficient is quasi constant during the experiment (i.e the temporal evolution of the HTC is negligible). In this communication we don't use this hypothesis since we determine the unsteady heat transfer coefficient over the all fin surface during the experiment delay.

\section{Experimental method description}

Figure 1 present the experimental test bench that allow the determination of temperature field on the second row of a circular fin tube heat exchanger model placed in the test section of a small wind tunnel. The air is extracted from a quiescent isothermal room through honeycomb and convergent. The air flow is achieved by a frequency controlled blower, placed at the end of the line, just after a flowmeter. Two synchronized solenoid valves (V1 and V2) set downstream the test section allow to bypass it or let the air flow passing through it. With this set-up, and using the procedure described in [2], [3], [4] the heat exchanger model is first heated using an infrared emitter and than cooled by forced convection. The HTC distribution on the fin surface is then deduced from the fin temperature field evolution measured by IR thermography using a Fluorine (CaF2) IR transparent window installed on the top of the test section.

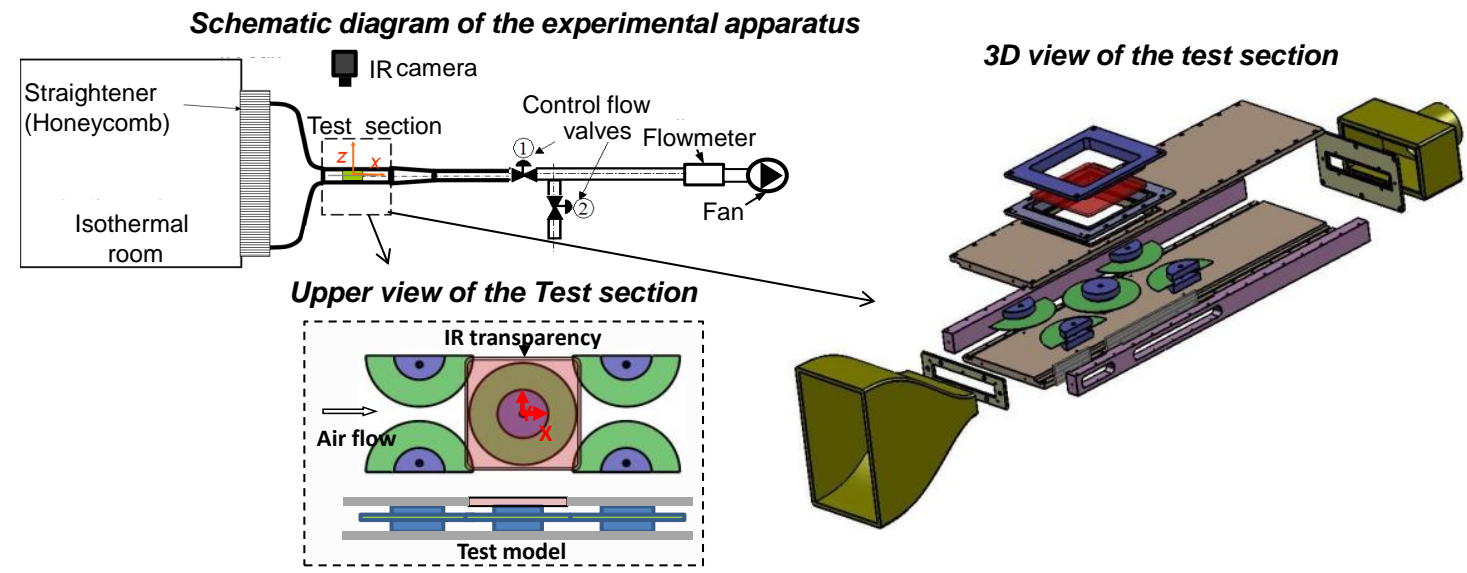

Fig. 1. Experimental test bench 


\section{Temperature measurements}

The IR set-up involves a Cedip Infrared® camera (Titanium 550M: InSb detector; 320x256 pixels; 3.6-5.1 $1 \mu \mathrm{m}$ ). The IR camera is calibrated in situ (see [3] for details) A special high conductivity plate (copper) coated with the same high emissivity coating as the fin, is placed in the test section. The calibration is made over the camera measurement range. The calibration law that links the object signal (in digital Level DL) recorded by the IR camera to the uniform temperature ( $\mathrm{T}$ in $\mathrm{K}$ ) of the calibration plate (given by a probe temperature), is a semi-empirical adaptation of Planck's law with three parameters $(R, B, F)$.

$$
D L=\frac{R}{e^{\frac{B}{T}}-F}
$$

The parameters values are calculated for the model by least-square fitting to the experimental points see figure 2(a).

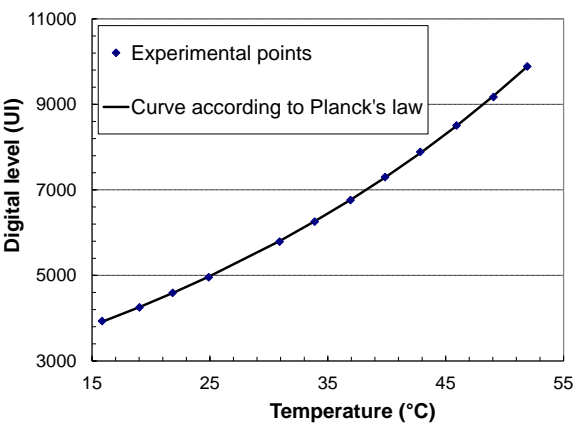

(a)

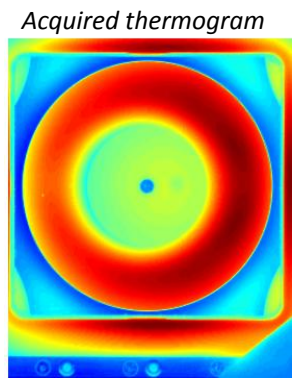

Fig. 2. Calibration curve and measurements treatment

The IR camera records the thermogram of the circular fin trough the infrared window at the different instants of the cooling. Figure 2(b) shows an example of thermogram acquired. After that, all pictures are cut out in order to save only the data corresponding to the circular fin zone. For that purpose, the Laplacian of the previous figure is performed. It allows easier edge detection in order to sort up the two circles that limit the region of interest. The two circles equations are determined using an algorithm that calculated the best least square fitting from 10 points picking up by the experimenter. A specific step is also made in order to remove erroneous data in images due to defects of camera detectors or specks of dust that may have fallen on the IR transparent window. The standard deviation of laplacian is calculated for each pixel. The pixels (and their neighbours) for which the Laplacian values are greater than 2.5 times the mean standard deviation are erased and recalculated by interpolation. Then, the calibrating function is used to convert the digital levels into temperatures to obtain the treated temperature field (figure 2(b)).

\section{Mathematical Formulation}

\subsection{The direct problem}

A specific algorithm, the direct problem, has been built in order to compute the time dependant fin temperature during the cooling period. The direct problem is based on a two dimensional approach (the fin is considered thermally thin) with a computational domain $\Omega$ presented in figure 3. The computational domain has two boundaries: the limiting circles, S1 and S2. The boundary condition are set to adiabatic and a uniform initial temperature is set on the medium for $\mathrm{t}=0$. The direct problem considered here is the determination of the temperature in a regular domain while thermal properties and boundary conditions are known. The direct problem can be expressed as follow:

$$
\begin{aligned}
& \rho \cdot C_{p} \cdot e \cdot \frac{\partial T(\Omega, t)}{\partial t}=\lambda \cdot e \cdot\left(\frac{\partial^{2} T(\Omega, t)}{\partial x^{2}}+\frac{\partial^{2} T(\Omega, t)}{\partial y^{2}}\right)-[\underbrace{h(\Omega, t) \cdot\left(T(\Omega, t)-T_{\text {ref }}\right)}_{\varphi_{g}(\Omega, t)}+\underbrace{\sigma \cdot \varepsilon\left(T^{4}(\Omega, t)-T_{\text {rad }}^{4}\right)}_{\varphi_{\text {conv }}}] \\
& \lambda \frac{\partial T\left(S_{i}, t\right)}{\partial n}=0 \quad i=1,2 \\
& T(\Omega, 0)=T_{\text {ini }}
\end{aligned}
$$


In equation 2 the term $e$ corresponds to half thickness of the fin. The solution is computed by expressing the boundary value problem of equation (2) in a weak formulation and by discretizing by the finite element method. The spatial domain is discretized using triangular elements (figure 3 ).

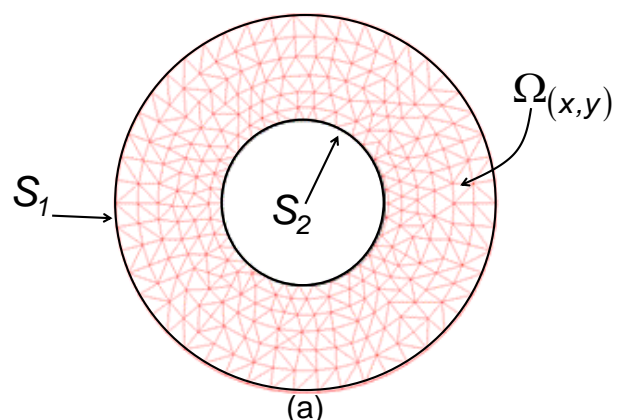

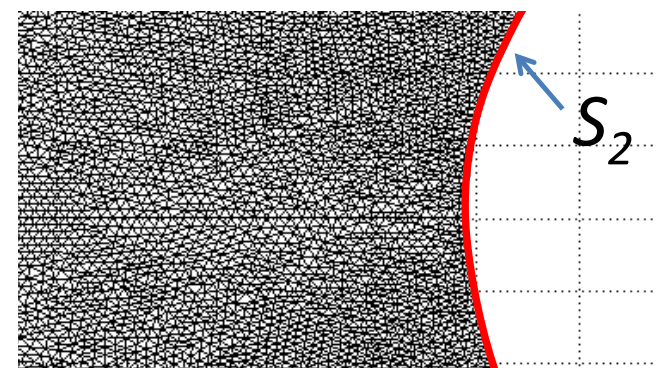

(b)

Fig. 3. (a) Computational domain and boundary condition (b) detail of the triangular mesh

On using the conventional finite element procedure the following matrix equation is obtained for the spatial discretization of the direct problem:

$$
\underline{\underline{\mathbf{M}}} \underline{T}^{\prime}+\underline{\mathbf{K}} \underline{\underline{T}}=\underline{F}
$$

With :

T $\operatorname{dim} N s$

vector of discrete temperatures function of time at the $N s$ discretization nodes

$T^{\prime} \quad \operatorname{dim} N s$

$\underline{\mathbf{M}} \quad \operatorname{dim}(\mathrm{Ns} \times \mathrm{Ns}) \quad$ mass matrix

$\stackrel{\mathbf{K}}{=} \quad \operatorname{dim}(N s \times N s) \quad$ stiffness matrix

F $\quad \operatorname{dim} N s \quad$ load vector function of the global heat flux in equation $2 \varphi_{g}(\Omega, t)$

With the load vector the direct problem allows the calculation of the temperature at each node of the fin. $\varphi_{g}$ is related to convection flux $\varphi_{\text {conv }}$ (that include the convection coefficient) and radiative flux $\varphi_{\text {rad }}$ :

$$
\varphi_{g}(\Omega, t)=\varphi_{\text {conv }}(\Omega, t)+\varphi_{\text {rad }}(\Omega, t)=h(\Omega, t) \cdot\left[T(\Omega, t)-T_{\text {ref }}\right]+\delta \sigma\left[T^{4}(\Omega, t)-T^{4}{ }_{\text {rad }}\right]
$$

In this last relation the vector $h$ is the vector of convection coefficient, function of time at the $N s$ discretisation nodes. The mesh is created using the freeware FREEFEM. On figure 3(a) the mesh is coarsen for better visualisation. A mesh sensitivity study has been conducted using the inverse algorithm presented hereafter. The study has shown that the solution is no more sensitive to the mesh density from a number of mesh elements of 130000 . This mesh density, presented in figure $3(\mathrm{~b})$ is used in the calculation performed for the circular fin and presented in section 6 .

For time discretisation of the problem, a Crank-Nicholson scheme is used. The matrix equation is then deduce from finite difference formulation in time :

$$
\left[\underline{\underline{\mathbf{M}}}+\frac{d t_{m}}{2} \underline{\underline{\mathbf{K}}}\right] \underline{T}_{m+1}=\left[\underline{\underline{\mathbf{M}}}-\frac{d t_{m}}{2} \underline{\underline{\mathbf{K}}}\right] \underline{T}_{m}+\frac{d t_{m}}{2}\left[\underline{F}_{m+1}+\underline{F}_{m}\right]
$$

This equation is resolved for the time indices $\mathrm{m}$ variable from 1 to $\mathrm{Nt}$ (number of time step) with the time step $d t_{m}$. At the first instant of the cooling the temperature field of the direct problem is set equal to the experimental temperature field.

\subsection{The inverse problem}

In the inverse problem the heat flux density $\varphi_{g}$ which is the input vector $\underline{\varphi}_{g}$, contained in the load vector $\underline{F}$ of the matrix equation of direct problem, is unknown. This vector is of size $N n=N s \cdot N t$. Nevertheless the experimental 
temperature field, measured during the cooling period, and the temperature field calculated with the direct problem for a given heat flux density are available. The inverse problem can so be express as a least square minimization problem of the measured and calculated temperature fields. The cost function $J=J\left(\underline{\varphi}_{g}\right)$ can so be formulated as :

$$
J=\frac{1}{2} \iint_{t}\left\|T(\Omega, t)-T^{*}(\Omega, t)\right\|^{2} d t d \Omega
$$

In this expression, $T(\Omega, t)$ is the measured temperature and $T^{*}(\Omega, t)$ the calculated temperature by the direct model based on a given or assumed vector. The integral over the time $t$ is made over $N t$ number of discrete time steps (directly link to the acquisition frequency of IR camera) during the period of cooling ranging between instants $\left[0, t_{f}\right]$. The integral over the domain $\Omega$ is made over $N$ s number of discrete nodes of the finite element mesh. Due to the important size of the computational domain, the large scale problem of searching vector $\varphi_{g}$ that minimize the cost function $J$ can be treated as a non linear optimization problem. The method uses to solve this non linear optimization is an iterative gradient based method known as the variable metric method (VMM) or quasi-Newton method $[5,6,7]$. The principle of the quasi-Newton method is to built from a first guess of unknown vector $\underline{\varphi}_{g}{ }^{0}$ a series defined by : $\underline{\varphi}_{g}{ }^{k+1}=\underline{\varphi}_{g}{ }^{k}+\beta^{k} d^{k}$. Where $k$ is the iteration index, $\beta^{k}$ is the step size and $d^{k}$ is the search direction. The search direction is computed from the cost function gradient and an approximation matrix $H^{k}$ to the inverse of the Hessian matrix :

$$
d^{k}=-H^{k} \nabla J\left(\underline{\varphi}{ }^{k}\right)
$$

In this last relation the matrix $H^{k}$ is a definite positive matrix, computed at each iteration of the process, initially set to the identity matrix. The new matrix at iteration $\mathrm{k}+1$ is calculated from information of preceding iteration $\mathrm{k}$ using the BFGS formula [8]. This iterative procedure is associated to an inexact line search of Armijo type for the evaluation of step size

$\beta^{k}$ [9]. In equation 7 the gradient $\nabla J\left(\underline{\varphi}_{g}{ }^{k}\right)$ is computed through the adjoint state method as described in [10].

The iterative process can be stated as follow:

Step 1: Start with initial guess for $\underline{\varphi}_{g}=>k=0 \quad \underline{\varphi}_{g}{ }^{k}=\underline{\varphi}_{g}{ }^{0}=0$ and with a $N n \times N n$ definite positive matrix $H$ : $H^{k}=H^{0}=I$ ( $/$ is the identity matrix).

Step 2: Solve the direct problem with $\underline{\varphi}_{g}{ }^{k}$ to obtain $\underline{T}(\Omega, t)$; evaluate the objective function $J^{k}=J\left(\underline{\varphi}_{g}{ }^{k}\right)$

Step 3 solve the adjoint problem to get the Lagrangian parameters ; calculate the gradient of the cost unction $\nabla J^{k}=\nabla J\left(\underline{\varphi}_{g}^{k}\right) ;$ calculate the norm of the gradient of the cost function $\left\|\nabla J^{k}\right\|$

Step 4: Check convergence using the two stopping criterion : test1 and test 2 . If the tests are satisfied the iterative process is stopped

Step 5: Calculation of search direction $d^{k}=-H^{k} \nabla J^{k}$; calculation of the optimal step size $\beta^{k}$. Calculate the new value of $\underline{\varphi}_{g}{ }^{k+1}=\underline{\varphi}_{g}{ }^{k}+\beta^{k} d^{k}$.

Step 6 Compute new estimation of $H^{k+1}$ from BFGS formula

Step 7 Go back to step 2

The stopping criterions use at step 4 are divided in two step : test 1 is that the cost function must be inferior to a small number: $J^{k}<\eta$. Test 2 is that the norm of the gradient of the cost function must also be inferior to a small number $\left\|\nabla J^{k}\right\|<\varepsilon$. For the first criterion a value of $\eta$ have to be set. The "Discrepancy Principle" [11] has been used in this study. In this method, the iterations are stopped when the residuals between measured and estimated temperatures are of the same order of magnitude of the measurement errors : $\left|T(\Omega, t)-T^{*}(\Omega, t)\right| \approx \sigma$. The value of $\eta$ is then equal to:

$$
\eta=\frac{1}{2} t_{f} \cdot S_{\Omega} \cdot \sigma^{2}
$$

With $t_{f}$ the cooling duration, $S_{\Omega}$. the surface of computational domain $\sigma$ the noise standard deviation. 


\section{5. validation of the inverse scheme - Inversion with simulated data}

In this part, the optimization and the quantification of the accuracy of the developed technique are performed using simulated measurements. In order to simplify the analysis the test case is monodimensional. It represents a thin monodimensionnal plate of thickness e with a boundary condition $\varphi_{\text {conv }}$ on its upper face and with adiabatic condition elsewhere (see figure 6(a)). Figure 6(b) and 6(c) presents the heat transfer coefficient distribution with its time variation uses to determined the boundary condition $\varphi_{\text {conv }}$ of the test case. Sharp variation in space and time are taken in order to test the stability of the inversion process. The spatio-temporal evolution of $h$ is determined by the product of the spatial distribution of $h$ (figure 6(b)) and its temporal variation (figure 6(c)). The convection coefficient distribution of figure 6 is used to calculate, using the direct model detailed in section 4.1, the time dependant temperature fields. These temperature fields are then used in the inversion scheme in order to retrieve the spatio-temporal distribution of $h$. To obtain more realistic data for testing the inverse scheme in the presence of noise, a random Gaussian noise is added with two values of noise amplitude, a small one with standard deviation of $0.03^{\circ} \mathrm{C}$ and a second one with standard deviation of $0.1^{\circ} \mathrm{C}$. Two values of conductivity and fin thickness of the fin are chosen in this test case. The first one is a relatively low conductivity case, (fin thickness of $0.5 \mathrm{~mm}$, conductivity equal $0.12 \mathrm{~W} \cdot \mathrm{m}^{-1} \cdot \mathrm{K}^{-1}$ ). This case corresponds to typical values taken by other researchers using transient method $[1,2,3,4]$. The second one is a relatively high conductivity case, (fin thickness equal to $0.21 \mathrm{~mm}$, conductivity equal $12 \mathrm{~W} \cdot \mathrm{m}^{-1} . \mathrm{K}^{-1}$ ). These two distinct values will help in the analysis of the influence of the diffusive fluxes in the inversion process. It is worth to note that for this two cases the Biot number remains always below 0.1 in order to respect the thermally thin hypothesis.

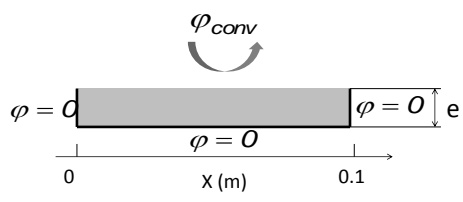

(a)

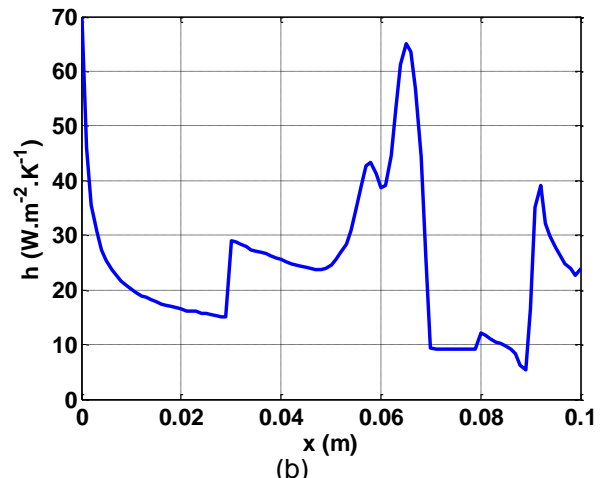

(b)

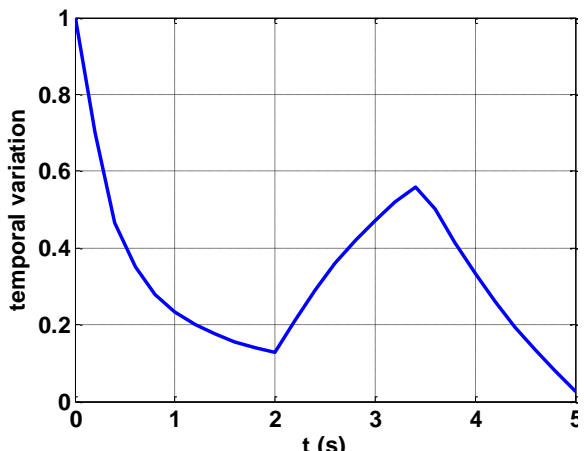

(c)

Fig. 6. Convection coefficient distribution in space and time of test case

The estimated heat transfer coefficient distributions determined with the inverse scheme are compared with the exact values on figure $7(\mathrm{a})$ and Figure $7(\mathrm{~b})$. Figure 7(a) presents the two cases of low and high conductivity cases and for the low value of noise. Figure 7 (b) presents the results for the high value of noise amplitude. The two figures are taken for the unique instant of cooling 3 second. From these two figures it is clear that the inversion process gives very good precisions. The estimated values of $h$ are very close to the exact ones even for the extreme case of high conductivity fin and high values of noise.

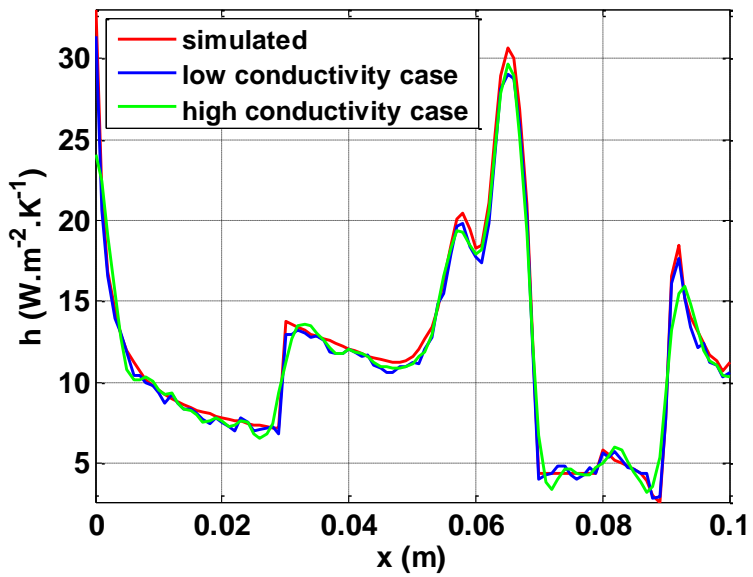

(a)

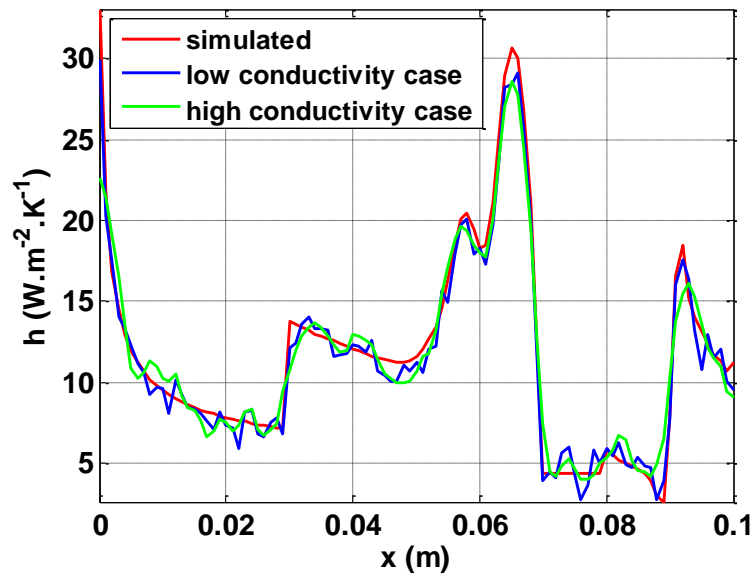

(b)

Fig. 7. $h$ distribution for the third second of the cooling (a) for the low noise data. (b) for the high noise data 


\section{Inversion with real experimental data}

The tests where performed on the circular fin. The fin is made of machinable ceramic with a fin thickness equal to $0.8 \mathrm{~mm}$ and a conductivity of $1.46 \mathrm{~W} \cdot \mathrm{m}^{-1} \cdot \mathrm{K}^{-1}$. This values set an experimental cases with relatively more important conductive fluxes inside the fin than other works $[1,2,3,4]$. The temperature fields are recorded during a cooling period of 5 seconds with an infrared camera frame rate equal to $100 \mathrm{Hertz}$. The inverse model described in section 4.2 use the measured temperature fields interpolated over the finite element mesh and uses one temperature field over 0.1 second (acquisition frequency of $10 \mathrm{~Hz}$ ) to reconstruct the spatio-temporal evolution of the convection coefficient during the cooling period of 5 seconds. Figure 8(a) and (b) present, as an example, the temperature field for the first and fifth second of cooling. At the same instants of the cooling Figure 8(c) (d) present the distribution of heat transfer coefficient calculated over the fin. In figure 8 the flow direction is from left to right. In accordance with previously published results $[1,2,3,4]$, heat transfer coefficient values are found to be high in the vicinity of the fin leading edge as well as upstream the tube. On the contrary, low values are noticed downstream the tube because of low velocities magnitudes that prevail in this wake zone. In the vicinity of the tube the Horse Shoe Vortex system with positive effect on heat transfer can be observed through high heat transfer coefficient values noticeable in a "U-shape" region wrapping round the tube. The HSV structure is found to be composed of three vortices for this value of Reynolds number.

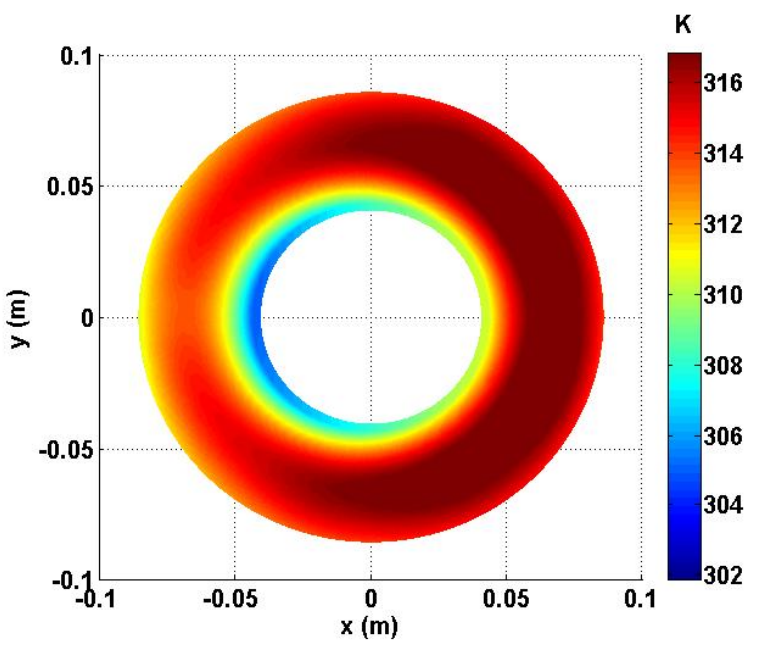

(a)

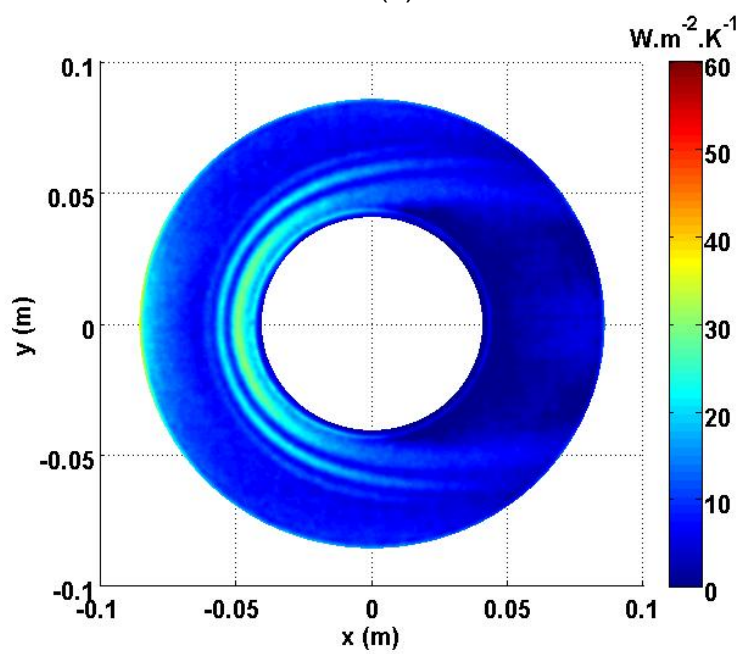

(c)

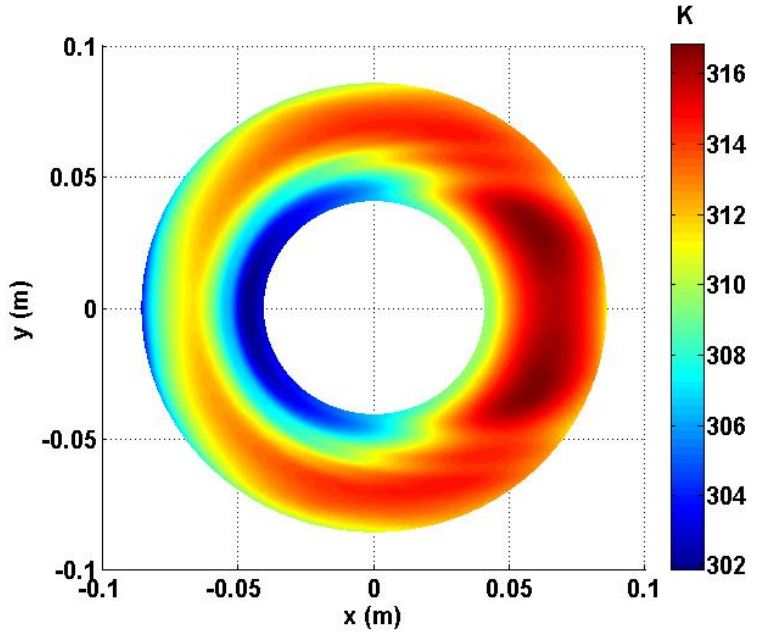

(b)

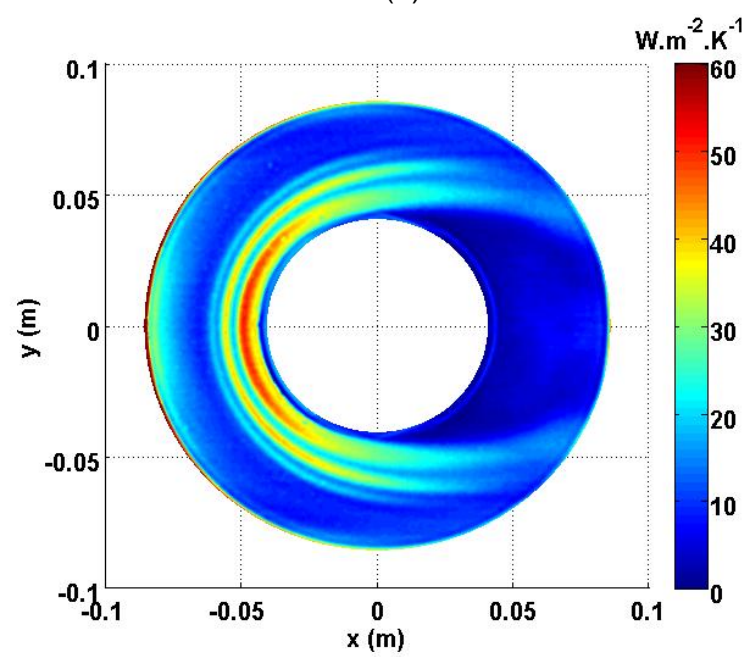

(d)

Fig. 8. Experimental temperature field at (a) 1 second and (b) 5 second of the cooling period and Heat transfer coefficient distribution at (c) 1 second and (d) 5 second of the cooling period

Concerning the temporal variation of the heat transfer coefficient, it is obvious from figure $8(\mathrm{c})$ and $8(\mathrm{~d})$ that the heat transfer coefficient undergoes a significant variation between these two instants. Figure 9(a) present the variation of the heat transfer coefficient, averaged over the entire fin, during all the cooling period. This instructive figure shows that there is an important variation during the first second of cooling. The averaged values of $h$ vary more than 8 percents (figure $9(\mathrm{~b})$ ). After this transient period the $h$ coefficient remains roughly constant (less than $1.5 \%$ of variation) until the fourth second where a slightly augmentation occurs. 


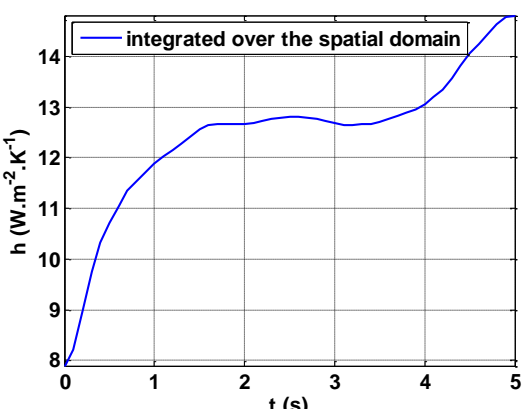

(a)

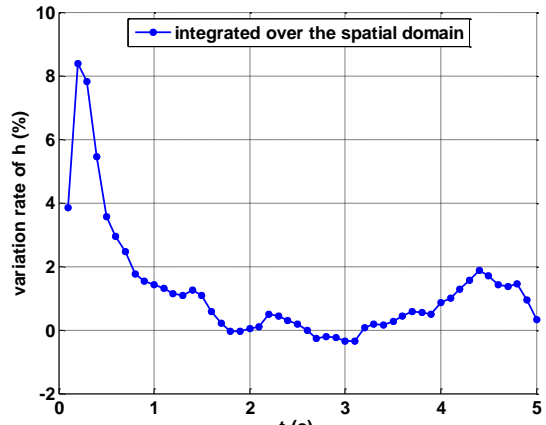

(b)

Fig. 9. (a) variation with time of the heat transfer coefficient integrated over the spatial domain (b) variation rate of the heat transfer coefficient integrated over the spatial domain

It is difficult to know the exact reasons of these variations. For the variation in the first second of the cooling, several reasons can be claimed: a physical inertia of the flow dynamics, an inertia time for the establishment of the thermal boundary layer structure or a too low values of thermal gradients (and drop of temperature) over the fin in respect with the noise values that impact the quality of the inversion process. Deeper investigations are needed to well understand this variation in the first second. For the variation after the fourth second, the problem seems to be that the thermal condition, which was close to a uniform temperature field over the fin, becomes more and more different with great spatial variation of temperature field. Hence regions appear over the fin where temperature values don't vary anymore versus time because they begin to reach the local value of the air temperature degrading convective flux calculation. Between the second and fourth second the average convection coefficient seem to reach a quite constant value.

On figure $10(\mathrm{~b})$ we have calculated the averaged heat transfer coefficient between the second and fourth second of cooling (with spatial averaged value of h quasi constant versus time). Figure 10(a) show the variation of this heat transfer coefficient around its local mean between the second and fourth seconds. It is obvious from this last figure that even if the heat transfer coefficient is globally constant between the second and fourth period (as already seen in figure 9) locally high variation can occurs. The first region of important variations is in the wake zone (downstream the tube). However in this region the heat transfer coefficient is almost zero, (with a poor precision of the determination of this coefficient by the inverse method), thereby reducing the significance of this slight temporal fluctuation. The second region is in the $U$ shape of the HSV thermal imprints. Clearly variation of about $30-50 \%$ is found very locally in the region of the thermal peaks of $h$ due to the HSV structure. This variation seems to show that the dynamic structure of the HSV varied with time. But this hypothesis is not totally convincing as the thermal imprints seen on figure 10(a) are thinner than the thermal imprints due to the vortices of HSV structure (see figure 10(b)) and outnumber the HSV vortices. An other explanation under investigation is that the localisations of vortices are not totally fixed during the cooling period creating these artefacts when calculating the change rate of local heat transfer coefficient.

$\delta \mathrm{h} w \cdot \mathrm{m}^{-2} \cdot \mathrm{K}^{-1}$

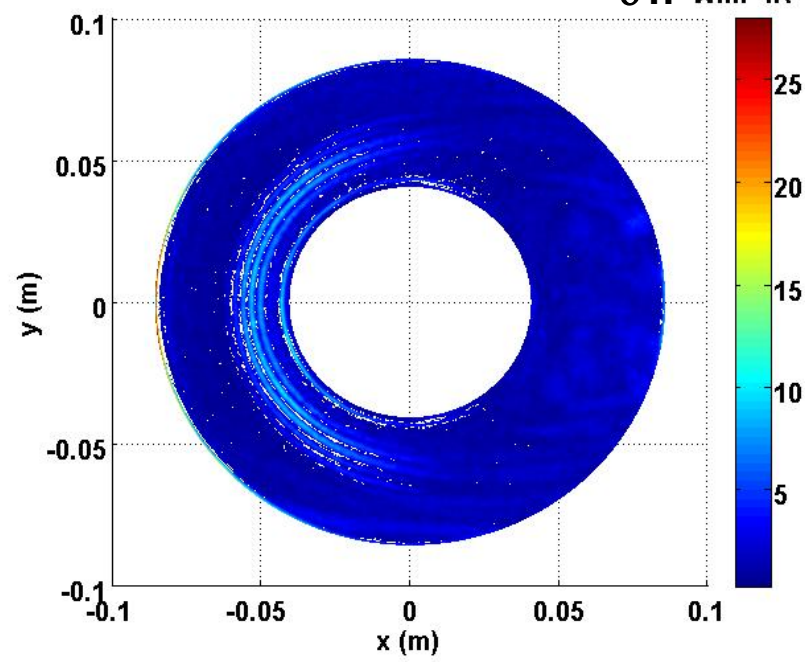

(a)

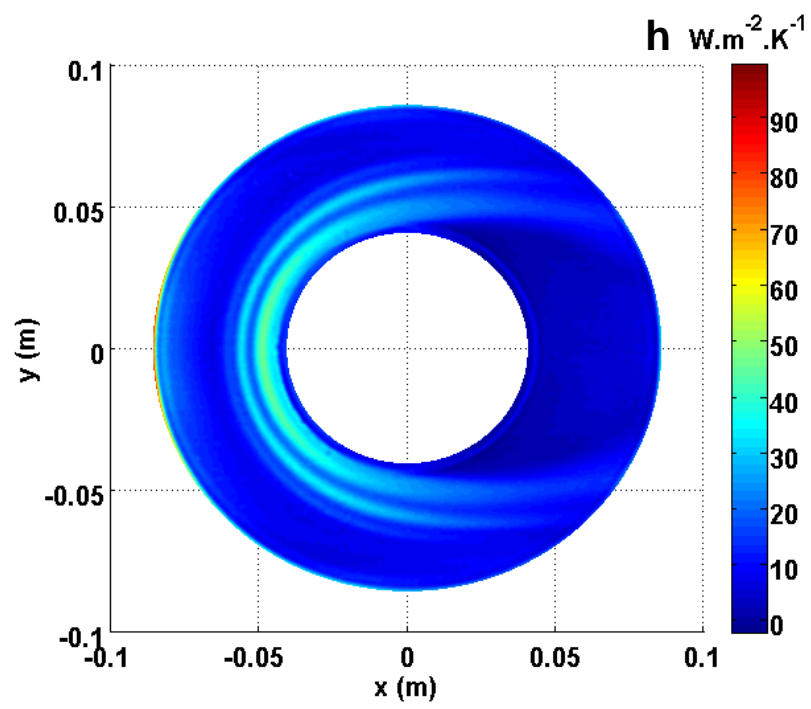

(b)

Fig. 10. (a) Change rate of the mean convection coefficient during the cooling (b) variation of the local heat transfer coefficient between the second and fourth seconds of cooling 


\section{Conclusions}

The study presented in this work concerns an inverse method used to determine the transient heat transfer coefficient distribution over the circular fin of a staggered finned tube heat exchanger assembly. The model is placed in a small wind tunnel and undergoes a transient cooling. The inverse method uses thermal fields acquired by an infrared camera during the cooling period to retrieve spatial heat flux distributions. The knowledge of these heat flux distributions and temperature distributions over the time period allows the calculation of the local heat transfer coefficient variation versus time. The direct problem algorithm use a finite element formulation in space for the computational domain, and a Crank-Nicholson scheme is used for time integration. The inverse problem is treated as a non linear optimization problem with an iterative gradient based method known as the variable metric method (VMM) or quasi-Newton method.

The efficiency of the method is test using simulated data and for different levels of noise and for different fin conductivities.

The method is then uses to determine the spatial and temporal evolution of the heat transfer coefficient during the transient technique. The spatial variation of the heat transfer coefficient is found to be close to preceding investigations using transient lumped capacitance method with regions of high variation of convective fluxes due to a complex fluid structure over the fin. The temporal evolution of the spatial averaged heat transfer coefficient is found to be low during the cooling period. Nevertheless locally in the region of Horse Shoe Vortex structure significant variations of local convection coefficient are found. Future investigations will allow us to better understand these variations.

The data provided by this new program will help better develop transient methods for determining the convective properties of different geometries. The method will allow in particular determining a relevant criterion for stopping the time integration in transient method.

\section{REFERENCES}

[1] Kim, Y. Y., Kim, K. S., Jeong, G. H., and Jeong, S., "An experimental study on the quantitative interpretation of local convective heat transfer for a plate fin and tube heat exchanger using the lumped capacitance method". International Journal of Heat and Mass Transfer, 49(1-2), pp. 230-239, 2006.

[2] Bougeard, D., "Infrared thermography investigation of local heat transfer in a plate fin and two-tube rows assembly". International Journal of Heat and Fluid Flow, 28(5), pp 988-1002.

[3] Vintrou S, Bougeard D, Russeil S, Nacerredine R, Harion J-L., "Quantitative infrared investigation of local heat transfer in a circular finned tube heat exchanger assembly". International Journal of Heat and Fluid Flow, vol. 44, pp 197-207, 2013.

[4] Vintrou S., Bougeard D., Russeil S., Nacereddine R., Harion J-L. "Infrared thermography for local Nusselt number estimation of an elliptical fin with a transient method", Proceedings of 11th Quantitative InfraRed Thermography conference, paper QIRT2012-139, Naples, (Italy), 2012

[5] Mobtil M., Bougeard D., "Identification du coefficient d'échange instationnaire par la méthode métrique variable associée au problème adjoint”, Congrès Français de Thermique, , Vol 1, pp 161-166, Le Touquet (France) 2010

[6] Kowsary F., Behbahaninia A., Pourshaghaphy A., "Transient heat flux function estimation utilizing the variable metric method". Int. com. in Heat and Mass Transfer vol. 33, pp. 800-810, 2000.

[7] Martinez J.M., "Practical quasi-Newton methods for solving non linear systems". Journal of computational and Applied Mathematics, vol. 124(1-2), pp. 97-121, 2000.

[8] Murea C.M., "The BFGS algorithm for a non linear least squares problem arising from blood flow in arteries". Computers and Mathematics with Applications, vol.49(2-3), pp. 171-186, 2005

[9] Shi Z-J., Shen J., "Memory gradient method with Goldstein line search". Computers and Mathematics with Applications, vol53(1),pp. 28-40, 2007.

[10] Yang G. Z., Zabaras N., "The adjoint Method for an Inverse Design Problem in the directional Solidification of Binary Alloys". Journal of Computational Physics, vol. 140(2), pp. 3082-3090, 2009.

[11] Morozov V.A., "Numerical realization of the optimal discrepancy method". USSR Computational Mathematics and mathematical Physics, vol. 16(6), pp. 194-198, 1976. 